\title{
GIANT-CELL TUMOR AND BROWN TUMOR IN PATIENTS WITH RENAL FAILURE AND SECONDARY HYPERPARATHYROIDISM
}

\author{
Ewa Krasuska-Sławińska', Bogumiła Koeber', Wioletta Jarmużek², Maciej Pronicki ${ }^{3}$, Michał Szałwiński ${ }^{4}$, \\ Dorota Olczak-Kowalczyk ${ }^{5}$
}

'Department of Paediatric Dental Surgery and Department of Paediatric Dentistry, The Children's Memorial Health Institute, Warsaw, Poland ${ }^{2}$ Department of Nephrology, Kidney Transplantation and Hypertension, The Children's Memorial Health Institute, Warsaw, Poland ${ }^{3}$ Department of Pathology, The Children's Memorial Health Institute, Warsaw, Poland

${ }^{4}$ Clinic for Cranio-Maxillofacial Surgery, Oral Surgery and Implantology, Medical University of Warsaw, Poland

${ }^{5}$ Department of Paediatric Dentistry, Medical University of Warsaw, Poland

\begin{abstract}
INTRODUCTION: Central giant-cell granuloma and brown tumor are intraosseous lesions, and their etiology is not fully known. They are commonly observed in maxillofacial region in mandibular and maxillary bone. A central giant-cell lesion is not considered to be a neoplasm. Lesions diagnosed in patients with hyperparathyroidism are called "brown tumors". Their development is associated with high serum levels of parathyroid hormone in the course of primary and secondary hyperparathyroidism that are complication of renal failure. Clinically central giant-cell granuloma and brown tumor manifest as painless bone protrusion covered with normal mucosa.

CASE PResentation: The objective of the study was to present diagnostic process and treatment of three pediatric patients with end-stage renal failure who developed brown tumors (two patients with secondary hyperparathyroidism) and central giant-cell granuloma (one patient without hyperparathyroidism) in bones of the facial skeleton. Surgical treatment was implemented in all patients.

Conclusions: When central giant-cell granulomas and brown tumors of the maxilla are suspected in patients at the developmental age, it is necessary to perform a computed tomography examination because lesions may not be always visible in radiological images. Surgical resection of a lesion, but without adjacent teeth combined with a treatment of secondary hyperparathyroidism, is an effective method of management.
\end{abstract}

KEY WORDS: central giant-cell lesion, CGCG, brown tumor, oral cavity.

J Stoma 2020; 73, 5: 276-282

DOI: https://doi.org/10.5114/jos.2020.100647

\section{INTRODUCTION}

Central giant-cell granulomas and brown tumors are rare and histologically similar bone lesions, which can be differentiated based on the patient's clinical data.
A central giant-cell granuloma (CGCG) is an intraosseous lesion, and its etiology is not fully known. It may be a bone repair response to a trauma or inflammation, or it may be a developmental lesion associated with a tooth eruption process. A possible correlation between CGCG
JOURNAL OF STOMATOLOGY CZASOPISMO STOMATOLOGICZNE

$\sqrt{P T S P}$
ADDRESS FOR CORRESPONDENCE: Ewa Krasuska-Sławińska, Department of Paediatric Dental Surgery and Department of Paediatric Dentistry, The Children's Memorial Health Institute, Al. Dzieci Polskich 20, 04-730 Warsaw, Poland, e-mail: ewa_krasuska@onet.eu

ReCeIved: 05.06.2020 • ACCePted: 08.10.2020 • Published: 30.10.2020 
and mutation in the $S H 3 B P 2$ gene has been reported, and this gene is responsible for the activity of osteoblasts and osteoclasts. According to the classification of the World Health Organization, CGCG is not considered a neoplasm despite its clinical similarities to neoplastic lesions [1].

With regard to localization, CGCG is most commonly observed in the bones of facial skeleton, more frequently in mandible than in maxilla $[2,3]$. It is significantly more rarely observed in other bones of the skull, axial skeleton, and long bones [2, 3]. CGCG is diagnosed in patients at various ages, most frequently in children and young adults [4]. Moreover, it is more common in women and according to some authors, it may be a result of a correlation between secretion of female hormones and disease development [5].

A clinical sign of CGCG is a growing protrusion, which is not painful, covered with normal mucous membrane, which may lead to facial asymmetry. The radiological manifestation of lesion is not unanimous. An oval single or multi-chamber transparent area that is surrounded by well- or poorly-developed osteosclerotic layer is most commonly observed.

There are two variants of central giant-cell granuloma: non-aggressive and aggressive $[2,3,6]$. The aggressive type is characterized by significant rapid growth, and it may be accompanied by pain or paraesthesia, resorption of root apices of the adjacent teeth, and perforation of the cortical plate. In this variant, there is also a significantly higher tendency for a recurrence (4-20\%) compared to the non-aggressive variant $[2,3,6]$. From histological point of view, a central giant-cell granuloma consists of the fibrotic tissue without any atypical signs, mononuclear inflammatory cells, giant polynuclear cells, and bone tissue with hemorrhagic foci [7].

Brown tumors are diagnosed in patients with hyperparathyroidism. Functional parathyroid disturbances caused by neoplastic proliferation (adenocarcinoma or parathyroid cancer) or secondary hyperparathyroidism being a complication of chronic renal failure may, contribute to the development of this tumor. The name "brown tumor" is associated with a macroscopic color of tissue specimen, and it is described as dark red to brown because of profuse bleeding and accumulation of hemosiderin deposits inside the tumor.

Development of brown tumors is associated with an excessive activity of osteoclasts and osteoblasts because of increased secretion of parathormone (PTH), resulting in bone remodeling, including osteolysis, osteogenesis, and fibrosis of the bone marrow cavity $[9,10]$. With regard to histopathological manifestation, giant-cell granulomas and brown tumors are similar, and therefore a differential diagnosis is recommended to perform calcium-phosphate metabolism assays to exclude parathyroidism [7, 10-12].

Treatment of a central giant-cell granuloma and brown tumor depend on their location, extent, grading, and patient's age. Surgical resection of a lesion, together with the teeth inside the mass, is a method of choice [7]. In patients at the developmental age, it is acceptable to leave the teeth adjacent to the lesion in case of a central giant-cell granuloma [6]. If the procedure is not radical, the risk of disease recurrence is estimated to be $4-20 \%$ [10].

Other methods of treatment include glucocorticosteroids, calcitonin, interferon alpha, and radiation therapy. Treatment options apart from surgical procedures are thought to be effective for small lesions that grow slowly. Large and painful lesions characterized by fast growth should be treated surgically [10].

The paper aims to present three patients with endstage renal failure who developed brown tumors (2 patients with secondary hyperparathyroidism) and central giant-cell granuloma (one patient without hyperparathyroidism) in bones of the facial skeleton.

\section{CASE PRESENTATION}

Three children ( 2 boys and 1 girl), under the care of the Department of Nephrology and Renal Transplantation, IPCZD (Children's Memorial Health Institute) were treated surgically due to lesions in the maxillary or mandibular bones at the Outpatient Clinic of Dental Surgery. The age of children at the time of diagnosis: 10 years and 9 months (the girl and the MK patient) and 15 years and 3 months (the KA patient). Both boys were kidney recipients (12 and 26 months, MK and $\mathrm{KA}$, respectively) due to end-stage renal failure caused by nephrotic syndrome (MK) and urinary tract defect (KA). The girl was currently receiving dialysis because of chronic end-stage renal failure from nephrolithiasis, and was being prepared for an organ transplantation. The patient had a ventriculoperitoneal pulsar valve due to hydrocephalus and had a surgery of myelomeningocele at L-S. Retrospective analysis of medical documentation was performed, and current and past methods of treatment (duration of hemodialysis, number of procedures per week, medications) were recorded. Blood tests were performed, and the following parameters were measured: red blood cells (RBC) count, hemoglobin, $\mathrm{PTH}$, serum calcium and phosphorus, creatinine, urea. Clinical dental examination as well as radiological diagnostic tests of lesions, including a panoramic radiograph and computed tomography of the facial skeleton were performed.

\section{RESULTS}

Despite treatment, all patients developed end-stage renal failure. The MK boy had been receiving peritoneal dialysis for 3 years, KA had been receiving hemodialysis 3 times a week for 5 years, and the girl had been receiving dialysis twice a week for 2 years and 4 months. Both male patients had been receiving cyclosporine A as 
TABLE 1. Laboratory test results of three patients

\begin{tabular}{|l|c|c|c|c|}
\hline Parameter & KZ (girl) & KA (boy) & 4.77 & Reference range \\
\hline RBC (M/ml) & 3.25 & 5.45 & 13.7 & $12.5-5.5$ \\
\hline Hemoglobin (g/l) & 8.5 & 14.7 & 39.8 & $37.0-43.0$ \\
\hline Hematocrit (\%) & 27 & 46.4 & 2.48 & $2.25-2.75$ \\
\hline Serum Ca (mmol/l) & 2.23 & 2.50 & 1.60 & $1.05-1.85$ \\
\hline Serum P (mmol/l) & 1.95 & 1.12 & 98.1 & $11-62$ \\
\hline PTH (pq/ml) & 1213 & 1550 & 0.50 & $0.06-1.00$ \\
\hline Creatinine (mg/dl) & 2.22 & 1.20 & 27.0 & $10-50$ \\
\hline Urea (mg/dl) & 62.1 & 67.2 & 180 & $100-550$ \\
\hline Alkaline phosphatase (U/l) & 752 & No data & \\
\hline
\end{tabular}

$R B C$ - red blood cells, Ca-calcium, $P$ - potassium, $P T H$ - parathormone

TABLE 2. Comparison of clinical and radiological symptoms associated with lesions diagnosed as brown tumors and central giant-cell granuloma in three patients

\begin{tabular}{|c|c|c|c|}
\hline Factor & KZ (girl) & KA (boy) & MK (boy) \\
\hline Location & Mandible near teeth 43-45 & $\begin{array}{l}\text { Maxilla near teeth } 16-18 \text {, } \\
\text { Mandible near teeth } 45-48\end{array}$ & Maxilla near teeth 22-23 \\
\hline Change of the facial features & No & Yes & Yes \\
\hline Intraoral symptoms & Elastic and soft protrusion & Hard protrusion & Elastic and soft protrusion \\
\hline Mucous membrane near the lesion & Normal & Normal & Normal \\
\hline Radiological image & $\begin{array}{l}\text { Oval depression with smooth } \\
\text { outlines in the alveolar part } \\
\text { of the mandible, } \\
\text { impacted teeth } 43,44,45\end{array}$ & $\begin{array}{l}\text { Presence of an oval transparent } \\
\text { area with an osteosclerotic layer in } \\
\text { the mandibular body impacted teeth } \\
47,48 \text {, supernumerary teeth near } 44\end{array}$ & Separation of roots of teeth 22,23 \\
\hline Tomography image & $\begin{array}{c}\text { Dimensions } 22 \times 20 \times 15 \mathrm{~mm} \\
\text { bone destruction with segmental } \\
\text { expansion }\end{array}$ & $\begin{array}{l}\text { Dimensions in the maxilla } 24 \times 36 \times \\
30 \mathrm{~mm} \text {, thickening, remodeling and } \\
\text { blurring of the normal bone structure } \\
\text { in the mandible, lesion diameter } \\
30 \mathrm{~mm} \text { osteolytic lesion without } \\
\text { a periosteal reaction }\end{array}$ & Dimensions $12 \times 9 \times 15 \mathrm{~mm}$ \\
\hline Histopathological diagnosis & Brown tumor & Brown tumor & Central giant-cell granuloma \\
\hline
\end{tabular}

a part of immunosuppressive treatment. In the younger boy (MK), cyclosporine A was replaced with tacrolimus 13 months after transplantation. The girl was on a kidney transplant waiting list. In two patients, secondary hyperparathyroidism developed very rapidly, while in one patient, the PTH levels were only slightly elevated $(98.1 \mathrm{pq} / \mathrm{ml})$. In this patient, secondary hyperparathyroidism was not diagnosed. Table 1 shows the results of laboratory tests in all patients.

Patients with secondary hyperparathyroidism received pharmacological treatment, including calcium carbonicum and $\alpha$-calcidol.

All patients were referred to the Outpatient Dental Clinic for Children due to pathological protrusions covered with intact mucous membrane in the maxilla (MK), mandible (girl), or both in the maxilla and mandible (KA). Table 2 indicates the comparison of clinical and radiological parameters of lesions observed.
An extraoral examination in male patients showed changes in facial features. The KA patient showed increased asymmetry and thickened facial features near the mandibular angle on the right and buccal area on the same side, while MK presented shallow philtrum and protrusion in this area on the left. There were no abnormalities in the extraoral examination in the girl. In the older boy (KA), the intraoral examination revealed hard and not painful protrusion of the alveolar part in the mandible on the right and alveolar process of the maxilla and hard palate on the right as well. In two other patients, the lesions were consistent with elastic and soft protrusion, causing shallowing of the vestibule and protrusion of the maxillary alveolar process near teeth 22 and 23 (patient MK), or mandibular body near the impacted teeth 44 and 45 . The submandibular lymph nodes were enlarged, single, and freely movable. Panoramic radiographs were taken in all patients. Only 

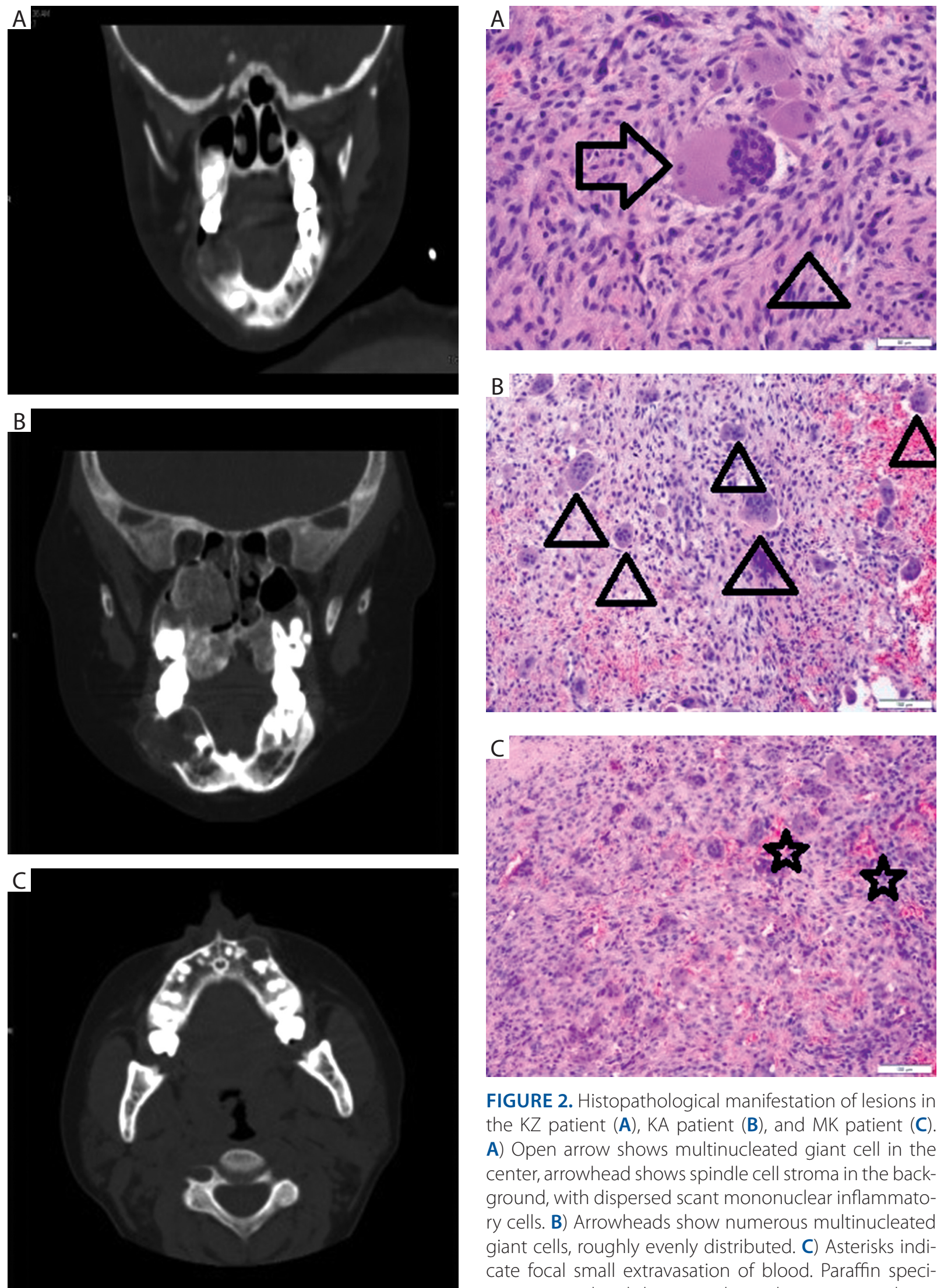

FIGURE 2. Histopathological manifestation of lesions in the $\mathrm{KZ}$ patient $(\mathbf{A}), \mathrm{KA}$ patient $(\mathbf{B})$, and MK patient $(\mathbf{C})$ A) Open arrow shows multinucleated giant cell in the center, arrowhead shows spindle cell stroma in the background, with dispersed scant mononuclear inflammatory cells. B) Arrowheads show numerous multinucleated giant cells, roughly evenly distributed. C) Asterisks indicate focal small extravasation of blood. Paraffin specimens stained with hematoxylin and eosin, original magnification: A) 400x; B, C) 200x

FIGURE 1. Computed tomography showing foci of low bone density located in the mandibular body on the right in the $\mathrm{KZ}$ patient $(\mathbf{A}), \mathrm{KA}$ patient $(\mathbf{B})$, and $\mathrm{MK}$ patient $(\mathbf{C})$ 
in the KA patient (older boy), a radiological image did show the presence of oval transparent area with an osteosclerotic layer in the mandibular body reaching from the impacted tooth 44 to the impacted tooth 47 . Normal trabeculation was observed in the maxilla. A panoramic radiograph of the girl showed impacted and abnormally located teeth 43 , 44, oval depression with smooth outlines in the alveolar part of the mandible in this area. With regard to the MK patient, only mild root deviation of teeth 22 and 23 was visible. Both in the girl and MK patient, the bone had normal trabeculation in the area corresponding to clinically observed abnormalities. In order to perform more detailed diagnostic tests in all patients, a computed tomography scan of the facial skeleton was performed, indicating bone destruction in the areas corresponding to clinical lesions (Figure 1).

A decision was made to implement surgical treatment in all patients. With regard to the female patient, a procedure of complete tumor resection was preceded by biopsy and histopathological examination. In the KA and MK patients, histopathological examination was performed after complete tumor resection. Samples were sent for histopathological examination. Taking into account only slightly elevated PTH levels $(98.1 \mathrm{pq} / \mathrm{ml})$ and lack of other symptoms of secondary hyperparathyroidism, the MK patient was diagnosed with a central giant-cell tumor, whereas considering high PTH levels (1550 pq/ml) and confirmed secondary hyperparathyroidism, the KA patient was diagnosed with a brown tumor (Figure 2).

In all patients, post-operative period was without complications, and they were regularly attending followup visits. Because of lack of any clinical symptoms indicating a recurrence, a follow-up computed tomography was not performed. However, dental images of the area from where tumors were removed, and panoramic radiographs were taken. The boys remain under the care of the Outpatient Clinic of Dental Surgery IPCZD (Child's Health Centre Institute). The time period since resection is $9(\mathrm{KA})$ and $7(\mathrm{MK})$ years, whereas 1.5 year after the resection of brown tumor, the girl died due to neurological complications associated with ventriculoperitoneal valve.

\section{DISCUSSION}

In literature, brown tumor lesions are relatively rarely described. Moreover, central giant-cell granuloma is not often observed in patients at the developmental age. Brown tumors are most frequently associated with endstage renal failure and secondary hyperparathyroidism. They are detected in $1.5-1.7 \%$ of patients with secondary hyperparathyroidism. Their development is correlated with high serum levels of PTH in the course of primary and secondary hyperparathyroidism, which is a complication of renal failure $[4,8,9,13]$. It is necessary to assess clinical symptoms, radiological signs, laboratory, and histology test results to differentiate a central giant-cell granuloma from a brown tumor, reparative granuloma, aneurysmal cyst, cherubism, Paget disease, and fibrous dysplasia [7, 10-12, 20]. Central giant-cell granuloma and brown tumor are mainly differentiated based on the presence of secondary hyperparathyroidism and laboratory tests for PTH, calcium, and phosphorus. Histopathological manifestation is not specific, and it has been emphasized by many authors. In all three patients presented herein, the histopathological manifestation of lesions was extremely similar (Figure 2). Diffuse polynuclear cells were visible in the spindle-cell stroma. In the background, there was extravasation and effusion of blood, and diffuse infiltration by mononuclear inflammatory cells. As it has been earlier mentioned, this manifestation is not specific, and it does not allow for differentiation of lesion character without considering the clinical background. It should be also emphasized that fields with a similar tissue are present inside classic giant-cell tumors, in the walls of aneurysmal cysts, and proliferative lesions, such as cherubism. Considering histopathologic findings, many differential diagnoses are sometimes possible, especially when small specimens are evaluated.

In the present paper, all patients suffered from endstage renal failure and in two of them, this condition was associated with secondary hyperparathyroidism and high levels of parathyroid hormone. The MK patient was not diagnosed with secondary hyperparathyroidism, and PTH levels were only slightly elevated. Kidney transplantation allows to balance calcium and phosphate metabolism disturbances, to reduce the parathyroid hormone levels, and to provide appropriate bone mineralization.

Both brown tumors and giant-cell granulomas may occur as diseases with one or more foci. They usually grow slowly, are not painful, or associated only with mild pain. With regard to palpation examination, they are mainly described as elastic and soft protrusions causing compact bone damage $[14,15]$. Large tumors may cause changes in the facial features and changes during tooth eruption [5]. In patients with a brown tumor and in case of the boy with a giant-cell granuloma presented here, the lesions are not painful and the external cortical plate of maxillary alveolar process and the alveolar part of mandible are damaged, which indicate an aggressive nature of the lesions. In the KA patient and the girl, the teeth were also abnormally located, and there were tooth eruption disorders.

According to Plechow et al., brown tumors visible on a radiological image are presented as osteolytic foci with one or more chambers, and they do not cause resorption of tooth roots adjacent to the tumor. Bodner et al. [16] reported bone destruction that has one or more chambers in radiological images (50/50\%), usually well-delineated, and in $40 \%$ of cases, there was a bone cortical layer at 
the border of the lesion, whereas in $60 \%$ of cases, lesion borders were uneven. They also indicated that in $80 \%$ of examined patients, there was a movement of tooth roots and in single cases, there was also their resorption. In radiological images of the girl and the MK boy, bone trabeculation in the areas near the tumors was not modified; only in the KA patient, there were changes in the bone structure visible in a panoramic radiograph. Radiological images of all patients revealed dental abnormalities related to separation of teeth roots (MK patient) or tooth eruption disturbances near the tumors (the girl and the KA boy). Bonet et al. as well as other authors [4] claimed that computed tomography is the most precise examination to visualize bone abnormalities. This examination was performed in all our patients, and only then was possible to see bone destruction in places corresponding to the tumor location in the girl and MK patient. However, regarding the KA patient, osteolytic lesions were reported in the mandible and a dense bone structure in the maxilla. Resorption of tooth roots adjacent to the tumor was not observed in any of our patients in radiological images.

Treatment of both brown tumors and giant-cell granulomas include, above all, a complete resection of lesion $[4,14]$. However, in patients at the developmental age, some authors select surgical treatment, which is the most saving [17-19]. Various authors [9] report regression of brown tumors after compensation of secondary hyperparathyroidism (normal levels of PTH, calcium, and phosphorus).

In all our patients, the treatment included surgical and complete tumor resection, along with teeth inside the tumor mass in two patients. A decision to leave the teeth was made only in the MK patient, as these teeth were normally erupted (second lateral incisor and canine), were located near to the lesion, and their roots were not resorbed. Such a management is allowed in patients at the developmental age [17-20].

Patients with secondary hyperparathyroidism require pharmacological treatment, which mainly includes a reduction of PTH levels. In case of patients with renal failure, phosphate-binding agents, active vitamin D preparations, and calcimimetics are used. In cases where PTH does not respond to treatment, parathyroidectomy is recommended $[7,9]$. Patients with secondary hyperparathyroidism presented in this paper received pharmacological treatment including agents that bind phosphates in the gastrointestinal tract (calcium carbonicum) and active vitamin $\mathrm{D}_{3}$ metabolites ( $\alpha$-calcidol).

\section{CONCLUSIONS}

When central giant-cell granulomas and brown tumors of the maxilla are suspected in patients at the developmental age, it is necessary to perform a computed tomography examination because lesions may not be always visible in radiological images. Surgical resection of a lesion, but without adjacent teeth combined with treatment of secondary hyperparathyroidism, is an effective method of management.

\section{CONFLICT OF INTEREST}

The authors declare no potential conflicts of interest with respect to the research, authorship, and/or publication of this article.

\section{References}

1. Jundt G. Central giant cell lesion. In: Barnes L, Eveson JW, Reichart P, Sidransky D (eds.). World Health Organization Classification of tumours pathology and genetics of head and neck tumours. Lyon: IARC Press; 2005, p. 324.

2. Kruse-Losler BK, Diallo R, Gaertner C, Mischke KL, Joos U, Kleinheinz J. Central giant cell granuloma of the jaws: a clinical, radiologic and histopathologic study of 26 cases. Oral Surg Oral Med Oral Pathol Oral Radiol Endod 2006; 103: 346-354.

3. Reddy V, Saxena S, Aggarwal P, Sharma P, Reddy M. Incidence of central giant cell granuloma of the jaws with clinical and histological confirmation: an archival study in Northern India. $\mathrm{Br}$ J Oral Maxillofac Surg 2012; 50: 668-672.

4. Theologie-Lygidakisa N, Telonaa P, Michail-Strantziab C, Iatroua I. Treatment of central giant-cell granulomas of the jaws in children: conservative or radical surgical approach? J Cranio-Maxillofac Surg 2011; 39: 639-644.

5. Tamrikulu R, Erol B, Yilmaz U, Yaman F, Atilgan S. Central giant cell lesions of the jaws in children - the review of 34 cases. Biotechnol Biotechnol Eq 2007; 21: 211-214.

6. Szporek B, Cieślik T. Treatment of central giant cell granuloma review of literature. Czas Stomatol 2007; 60: 179-185 [In Polish].

7. Janas A. Ziarniniak olbrzymiokomórkowy. Dental Forum 2007; 35: 69-73.

8. Wartacz J, Konieczny A, Hałoń A, et al. Brown tumor in patient with chronic renal failure as a rare complication of the secondary hyperparathyroidism. Nefrol Dial Pol 2013; 17: 92-94 [In Polish].

9. Medeiros Queiroz S, Gadelha Vasconcelo R, de Andrade ALDL, et al. Maxillary brown tumor associated with chronic kidney failure: a case report. J Bras Patol Med Lab 2013; 49.

10. Ciorba A, Altissimi G, Giansanti M. Giant cell granuloma of the maxilla: case report. Acta Otorhinolaryngol Ital 2004; 24: 26-29.

11. Olczak-Kowalczyk D, Kosiorowska-Bednarczyk A, Stopa Z, et al. Brown tumor of the mandible and the maxilla in association with secondary hyperparathyroidism in patient after renal transplantation- own obserwations. Czas Stomatol 2009; 62: 816-823 [In Polish].

12. Zielińska-Kaźmierska B, Grodecka J, Neskromna A, Kobos J. Centra giant cell granuloma of the mandible in different clinical aspect: a rewiev of literature and own obserwation. Współcz Onkol 2006; 5: 250-254 [In Polish].

13. Pechalova P.F, Poriazova E.G. Brown tumor at the jaw in patients with secondary hyperparathyroidism due to chronic renal failure. Acta Medic 2013; 56: 83-86.

14. Adornato MC, Paticoff KA. Intralesional corticosteroid injection for treatment of central giant cell granuloma. J Am Dent Assoc 2001; 132: 186-190.

15. Allen DT, Sheats RD. A central giant cell granuloma in a patient seeking orthodontic treatment J Am Dent Assoc 2001; 132: 1255-1260.

16. Bodnet I, Bar-Ziv J. Radiographic features of central giant cel granulloma of the jaws in children. Pediatr Radiol 1996; 26: 148-151. 
17. de Lange J, van der Akker HP, van der Berg H. Central giant cel granuloma of the jaw: the rewiew of the literature with emphasis of therapy options. Oral Surg Oral Med Oral Pathol Oral Radiol Endod 2007; 104: 603-615.

18. Bathelemy I, Mondie J. Giant cell tumors and pseudogiant cell tumors of the jaw. Rev Stomatolog Chir Maxillofac 2009; 110: 209-213.

19. Tosco P, Tanteri G, Iaquinta C, et al. Surgical treatment and reconstruction for central giant cell granuloma of the jaw: a rewiew of 18 cases. J Cranio Maxillofac Surg 2009; 37: 380-387.

20. Krasuska-Sławińska E, Świstak K, Jarmużek W, Pronicki M, Olczak-Kowalczyk D. Central giant cel granuloma in 11-years old boy after kidney transplantation. Nowa Stomatol 2012; 4: 152-155 [In Polish]. 